à tension croissant avec la charge du réseau. Ge compoundage peut être obtenu, soit mécaniquement, soit par un dispositif électrique comportant entre-aiutres un transformateur d'intensité mesurant le débit de la ligne.

Le régulateur Guénod-R.S. est construit en différents modèles selon la puissance du réseau et peut corriger toutes les variations de tension, quelles que soient leur grandeur et leur répartition en plus ou en moins de la valeur de consigne.

Genre de montage. - L'appareil est livrable dans les 3 exécutions suivantes :

Pour montage dans cabine, derrière tablean, sans protection (fig. 2).

Pour montage dans un local, l'appareil possédant une protection en tòle perforée.

Pour montage sur potea'u, l'appareil fixé dans une coquille munie du dispositif pour fixation contre un poteau (fig. 3).

Pour toute demande, on spécifiera dans chaque cas :

$1^{\circ}$ Le genre de courant et la fréquence du réseau à régler.

$2^{\circ}$ La tension à maintenir constante.

$3^{\circ}$ Les variations de tension à corriger.

$4^{\circ}$ L'intensité maximum de passage.

$5^{\circ}$ Les dispositions spéciales éventuelles.

Avantages du régulateur. - Le régulateur type R.S. est, en somme, un régulateur d'induction automatique dont la construction est étudiée pour obtenir un prix de vente admissible, même dans les petites installations. L'appareil possède donc tous les avantages bien connus du régulateur d'induction par rapport aux transformateurs à gradins ou autres, avantages résumés plus loin, mais il présente encore la particularité intéressante d'un décalage pratiquement nul.

Le régulateur type R.S. règle d'une façon progressive permanente, sans à-coup de tension; il ne provoque donc aucun saut de lumière et aucun parasite en T.S.F., points dont l'importance n'échappera à personne ; l'organe de ré- glage n'a pas d'usure puisqu'il n'existe aucun contact, frotteur, roulette ou disposilif de ce genre. Il ne se produit aucune coupure de courant dans le réseau. L'appareil n'est

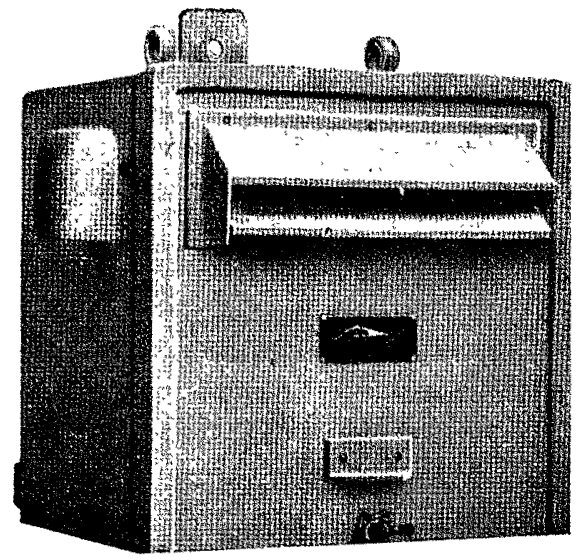

Fig. 3

Type RSp pour montage sur poteaux.

pas temporisé comme cela est nécessaire pour éviter un travail trop intensif dans les systèmes à commutateur.

Le régulateur Guénod lype R.S. ne demande pas d'entretien, en particulier, aucun renouvellement de contacts, ni limitation du nombre d'enclenchements. Son fonctionncment est silencieux comparablle à celui des transformateurs. Enfin, sa sécurité de fonctionnement est absolue car, non seulement l'ongane de réglage lui-même, mais aussi toutes les autres parties ont été étudiées pour assurer à l'exploitation le maximum de sécurité. Les enroulements de réglage supportent sans dommage le court-circuit ; le moteur de commande à cage d'écureuil ne possède aucune pièce susceptible d'usure ou de détérioration ; enfin, tout l'appareil, conforme aux normes d'essais les plus récentes, est construit, par Ides ateliers spécialisés depuis 1899 dans l'exécution des régulateurs automatiques et de leurs accessoires.

\title{
Collaboration de la houille blanche et de la houille noire sur le réseau du Midi
}

\author{
par V. Charrin, Ingénieur-conseil
}

Un des meilleurs exemples du complément que peut apporter la houille noire à l'énergie hydroélectrique, nous est fourni par la Cie des Chemins de Fer du Midi.

Ce réseau a depuis longtemps poursuivi activement l'électrification de ses lignes; celle de Villefranche-Bourg-Madame date de 1911 et, l'an dernier, 1935, a été mis en service le tronçon Montauban-Sète, portant à 1863 kilomètres la totalité des voies électrifiées de ce réseau.

Il ne reste plus, en somme que le tronçon Montauban-Bordeaux pour que la traction électrique desserve tous les points principaux du Sud-Ouest.

La compagnie des Chemins de Fer du Midi produit ellemême la totallité du courant nécessaire à son exploitation : jusqu'en 1932, ce courant était d'origine exclusivement hydraulique ; aujourd'hui, le courant d'origine thermique devient quantité non négligeable, comme nous le verrons plus loin, ce n'est pas la nécessité qui a fait adopter cotte dualité de production, l'équipement des usines hydroélectriques permettant de fournir beaucoup plus que les besoins actuels ; c'est plutôt la proximité de la production thermique du gros nœud que va devenir Bordeaux et aussi, il faut le reconnaître, la possibilité de développemenl donnée ainsi à un bassin lignitifère jusque là considéré sans valeur.

Le courant électrique du Midi provient de trois groupes différents d'usines hydroélectriques :

$1^{\circ}$ Les deux usines de la Têt, dans les Pyrénécs-Orientales;

$2^{\circ}$ Les deux usines de Soulom, sur les gaves de Pau et de Cauteret, et d'Eget, sur la Nesle, dans les Pyrénées centrales; 
$3^{\circ}$ Les trois usines du Gave d'Ossau, dans les Basses-Pyrénées.

Les usines des Pyrénées-Orientales, installées toutes deux sur la Têt, sont respectivement de 4.000 et $2.500 \mathrm{KW}$; elles servent uniquement à alimenter les lignes de Villefranche à la Tour-de-Carol et de Perpignan à Villefranche, en courant triphasé à 25 périodes et en courant monophasé à 16 périodes.

L'équipement des Pyrénées Centrales est beaucoup plus important ; il est de $15.000 \mathrm{KW}$ pour l'usine de Soulom ct de 25.000 pour celle d'Eget ; cette dernière est alimentée par les lacs d'Aumar, d'A'ubert, de Cap de Long, d'Orédon et par le réservoir nrtificiel de l'Oule, dont la capacité totale dépasse 26 millions de mètres cubes.

Quant aux usines de la vallées d'Ossau, elles sont disposées en cascade an-dessus de Laruns ; celle d'Artouste, qui utilise les eaux du lac de même nom, dispose d'une chute de $765 \mathrm{~m}$.: l'usine de Miegebat, qui se trouve à l'aval de la précédente, reçoit l'eau sous 390 mètres et l'usine du Houbat, encore plus à l'aval, sous 200 mètres. Leur équipement total atteint $90.000 \mathrm{KW}$.

Cies cinq dernières usines fournissent le courant triphasé ì la fréquence de 50 périodes et à la tension de 60.000 volts, qui est envoyé aux sous-stations dispersées sur tout le réseau. Quand la distance de transport dépasse 300 kilomètres, la tension est portée à 150.000 volts.

Pour l'utilisation, le courant triphasé est transformé en courant continu de 1.500 volts ; les sous-stations sont espacées en moyenne, de 20 à 25 kilomètres.

On dispose ainsi dans les usines dont nous venons de parler d'environ 400 millions de kilowalts-heure annuels, tandis que la consommation est actuellement de l'ordre de 250 millions.

Depuis 1932, fonctionne à la mine d'Hostens, au Sud du département de la Gironde, appartenant à la Société Minière et électrique des Landes, une centrale de $25.000 \mathrm{KW}$ de puissance installée, qui livre la totalité de son courant à la Cie des Chemins de Fer du Midi.

Cette centrale comprend 2 groupes turbo-alternateurs de $12.500 \mathrm{KW}$, produisant du courant à 6.000 volts et alimentés par trois chaudières de $720 \mathrm{~m}^{2}$ et timbrées à 22 kilos. Elle est placée à quelques centaines de mètres de la mine de lignite d'Hostens, où une couche épaisse de 5 à $12 \mathrm{~m}$., recouverte par 2 à 3 mètres de sables, est exploitée à ciel ouvert.

Comme ce lignite est très spécial, qu'il renferme de 50 ì $60 \%$ d'eau et n'a jamais pu être utilisé auparavant - son pouvoir calorifique, à l'état brut, ne dépasse pas 1.500 calories - il fallait, avant tout, un prix de revient extrêmement bas. Le problème a été résolu au moven d'un portique d'extraction de 80 mètres de portée qui, par des pelles mécaniques, permet une production de 400 mètres cubes à l'heure, aveo un personnel extrêmement réduit.

Cette installation qui fait le plus grand honneur à la Société Minière et électrique des Landes, montre que ce vaste bassin lignitifère landais qui renferme plusieurs milliards de tonnes de charbon, est parfaitement exploitable quand on sait utiliser rationnellement ses produits.

La centrale est relliée au poste de Pessac près Bordeaux, de la $\mathrm{Cie}$ du Midi, par une ligne à 60.000 volts.

A ce poste se marient les courants électriques de la houille noire landaise et de la houille blanche pyrénéenne.

\section{DOCUMENTATION}

\section{La transformation des groupes électrogènes de l'usine hydro-électrique de Cusset-Villeurbanne de la Société Lyonnaise des Forces Motrices du Rhône}

Les progrès de la technique des turbines hydrauliques, en particulier en ce qui concernc le type hélice, sont tels que bien souvent on a intérêt à remplacer d'anciens groupes beaucoup plus puissants sous un même volume. Mais la transformation des fondations représente un travail tel, qu'il ne doit ôtre entrepris qu'avec circonspection.

C'est ainsi que la centrale de Cusset de la Société des Forces Mo. trices du Rhône est en cours de transformation. Cette usine, construite entre 1894 et 1897 est du type usine-barragc, situé à l'extrémité du Canal de Jonage.

La chute utile varie de 10 à $12 \mathrm{~m}$. Le débit dérivé est compris entre 100 et $150 \mathrm{~m}^{3}$. Le bâtiment comprend deux étages : l'étage supérieur est un hall de $152 \mathrm{~m}$. où se trouvent les alternateurs : l'étage inféricur est ríservé aux turbines.

Chaque turbine a son pertuis d'entrée d'cau, sa chambre et son canal de fuite. Le nombre de ces sortes de cellules hydrauliques est de 19, dont 16 occupées par les groupes électrogènes fournissant le courant alternatif triphasé distribué et las 3 autres par des groupes dynamos, produisant le courant continu d'excitation.

En première étapo furent installés 8 turbines Jonval, développant chacune $1.200 \mathrm{CV}$ à 120 tours par minute. Files étaient tronconiques centripètes, à 3 étages d'aubes, munies chacune d'un vannage cylindrique. Ioss deux élages inférieurs étaient manœurrés simultanémént par le régulateur, maintenant une vitesse constante à $3 \%$ près. Te vannage supérieur n'était utilisé qu'en temps de crue et pouvait être accouplé aux deux autres. Le rendement de ces turbines ne dépassait pas $67 \%$.

En deuxièmé étape on installa 8 turbines Francis doubles, d'une puissance de $1.350 \mathrm{CV}$. Elles avaient 2 roues motrices, l'une an dessus de l'autre, dans une huche en fonte. Le distributeur circulaire à aubes mobiles était commandé par un régulateur, assurant une précision de $0,25 \%$. Le rendement de ces turbines atteint $75 \%$.

Les alternateurs, tous identiques, à axe vertical, sont du typc ombrelle. Les inducteurs portent 50 pôles massifs. Leur pussance est de $1.350 \mathrm{KVA}$, sous 3.500 volts.

En 1917 et 1918 les roues conique's des turbines Jonval ont été remplacées par des roues Francis simples. La substitution, qui a porté simplement sur les roues motrices ct leur vannage, a procuré un gain de 12 à $15 \%$ tant sur le rendement que sur la puissance.

Parallèlement, les alternateurs ont été rebobinés et leur puissance est montée à $1.750 \mathrm{KVA}$, avec un rendement de $96 \%$.

En 1932, la Société Lyonnaise des Forces Motrices du Rhône a obtenu l'autorisation de construire un barrage mobile sur le Rhône, à $100 \mathrm{~m}$. en aval de l'entrée du Canal de Jonage, ce qui a permis d'élever de $0 \mathrm{~m}$. 50, le plan d'eau en amont de l'usine-barrage et d'assurer un débit pouvant atteindre $500 \mathrm{~m}^{3}$. La chute utile se trouve portée entre 11 et $13 \mathrm{~m}$.

Cette société a entrepris alors de remplacer ses anciens groupes par des groupes modernes 5 fois plus puissants. Ceux-ci comprennent une turbine Kaplan à axe vertical tournant à $21 / 4$ tours par 